\title{
Research on the Sunlight Sports and Corresponding Involved Medical Problems and Sports Health Knowledge
}

\author{
Shanguo Liu \\ Chang Sha Medical University, \\ Changsha,Hunan,410219 China
}

\author{
Yue Wu \\ Chang Sha Medical University, \\ Changsha,Hunan,410219 China
}

\begin{abstract}
In this paper, we conduct research on the sunlight sports and the corresponding involved medical problems and the sports health knowledge. Life lies in movement, and movement in the correlation of constitution health and physical exercise are presented, embodies the physical exercise behavior and the importance of play to students' physique health influence and the role of physical training on college students' physical health, increasing importance for the work of colleges and universities sports education and reform, in the existing school education for college students to participate in the condition of physical exercise, to explore the effective method to improve students' physique healthy exercise or approach, not only to cultivate the students' consciousness of physical exercise, physical exercise behavior ability, lifelong exercise habit is particularly important, and for colleges and universities sports education work has more profound significance. Our research serves as the new paradigm for education which is meaningful.
\end{abstract}

\section{Keywords-Sunlight Sports, Involved Medical, Sports Health, Problems, Knowledge.}

\section{Introduction}

Physical exercise behavior problems research belongs to the basic category of the emerging physical exercise psychology. Overall, physical exercise psychology research history is shorter, about in the late $1980 \mathrm{~s}$ began to study abroad. From the point of the existing data, the current domestic in sports psychology research on physical exercise behavior is relatively small, the research methods are single. Especially the research on physical exercise behavior of college students is not much in one of the few in the study of physical exercise behavior of college students still has some limitations.

Along with the development of society and human progress, people gradually increasing awareness of health, at the same time also more and more realize the importance of the physical exercise on body health by many studies have shown that physical health and physical exercise of correlation, to reflect the physical exercise behavior for the importance of college students physical health but for now this special group in college students, in physical exercise participation of the cognitive and behavioral deviation phenomenon exists obvious separation and university stage is a special and critical stage, as the college students at this stage the understanding of physical exercise, exercise behavior habits of consciousness, in the future, to be going to work to maintain a healthy body and lifelong participation in physical exercise are relatively positive impact [1-2].

The role of physical health in school sports could be generally summarized as follows. (1) Prevent the happening of the sports injury. In physical training, the occurrence of sports injury is often all of a sudden, the incident has unpredictability. Have the certain physical health knowledge to reduce the incidence of sports injury, sports injury prevention techniques in basic movement, error, inadequate preparation and ego to protect consciousness is not high and uneven sports venues are the factors lead to 
injury. (2) To improve the students' sports scientific knowledge reserves. Life lies in the scientific movement. Science movement not only refers to the reasonable control of exercise intensity, exercise, and also including the sports injury prevention, scientific hydrating and sports nutrition knowledge. (3) To promote the formation of students' lifelong sports view. School students are in the peak period of growth and development as also is the outlook on life and values formation, in terms of ideology to have very strong plasticity. In the school sports teaching, in addition to teach students basic sports skills, physical health knowledge transmission is particularly important.

As one of the important means to promote the mental health of the physical exercise, also more and more get people's attention, studies the physical exercise of the mental health effects has become now and even after the international sport psychology and the key research topic in the field of the health psychology. In our country, the research work has just started and most of the research is still in a summary of the experience. The figure one shows the basic forms of exercises.

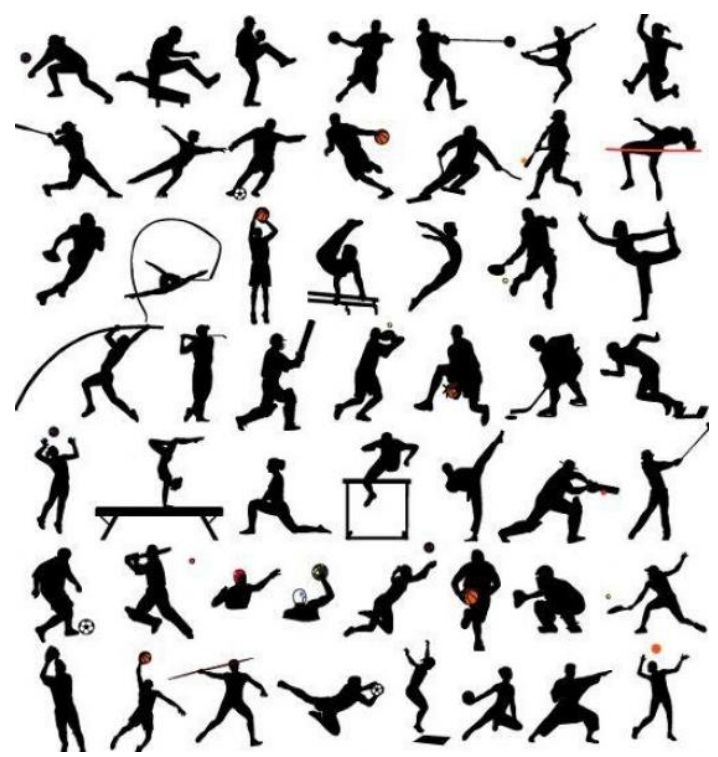

Figure 1. The Demonstration of the Basic Physical Exercise Activities
In this paper, we conduct research on the sunlight sports and the corresponding involved medical problems and sports health knowledge. The study found that regular physical exercise of the college students group, stress reaction significantly below do not often take exercise of college students. And studies have shown that consciously and actively exercise can accelerate the elimination of the mental fatigue, if keep a good mood and keep activity of moderate intensity, can reduce fatigue, through physical exercise can improve the physiological function such as maximum muscle strength to reduce the occurrence of fatigue. Later sections will discuss the issues in detail.

\section{Our Proposed Methodology}

The Sunlight Sports. The so-called sunshine sports movement, it is to point to by the ministry of the education, state general administration of sports, and the communist youth league central common decision, starting in the 2007, combined with the full implementation of the student physique healthy standard, extensive and in-depth in schools of various levels in the country to carry out the national hundreds of millions of students to referred to as "the sunlight sports. From the composition of the sunlight sports level, the sunlight sports from three aspects of the lack of material, behavior and the spiritual elements. Sunshine sports and college sports teaching reform in the related research could be generally organized as the following aspects.

- The meaning of sunshine sports in colleges and universities sports teaching content reform. Sunshine sports concept to attach great importance to students' health of body and the mind development, according to the students' physical and mental development characteristics to the differentiate students' learning level, and then set learning goals. The sunlight sports is education for the students, namely guarantee every student to enjoy 
equal rights of education, meet the development needs of the students as much as possible [3-4].

- The meaning of sunshine sports in colleges and universities sports teaching evaluation. Under the aim of sunshine sports, the sports examination system cannot meet the requirements of the now that need to set up scientific sports performance appraisal evaluation system.

- The meaning of sunshine sports in colleges and universities sports teaching goal. Adhere to the guiding ideology of the health first sunshine sports concept, physical development and the psychological development and social adaptation of three-dimensional health perspectives. According to the physical conditions of students' age, gender, and, actively explore to adapt to the characteristics of youth sports teaching method and activity form, guide students to carry out the plan that have purpose, regular physical exercise, efforts to improve the students' body form and function, improve sports ability and the physical health standard.

The sunlight sport is asking the country to hundreds of millions of teenager involved huge activity and it covers the scope of the great, involving the levels of the schools. Therefore, it will drive up the students' self-training that establishes the idea of health first. The school sports is the main in sunshine sports space, is the foundation of this activity. We should seize the opportunity, allow for the ascent of the social awareness of the school sports work height, it will be good for school sports work and the students' physical quality improvement [5].

The Principles of the Physical Exercise. Promote the development of the college students' mental health that is an important content of quality education, at the request of the quality education, health education problem is becoming more and more important in the school. The development of the society, people face many psychological pressures and crisis, also affects the healthy development of students' personality. The current situation of college students' mental health is not optimistic, with different degree of psychological barriers and the number of potential problems is on the rise, to the school education and management work has brought certain difficulty. Focusing on the analysis of college students' mental health related environment, explore the physical education activities, and the given priority to development, preventive, to enhance college students' life stress and psychological endurance of the setbacks, encourage students' psychology and behavior be enhanced through sports, especially through appropriate physical exercise behavior to develop mental health level of college students becomes more important and urgent. 


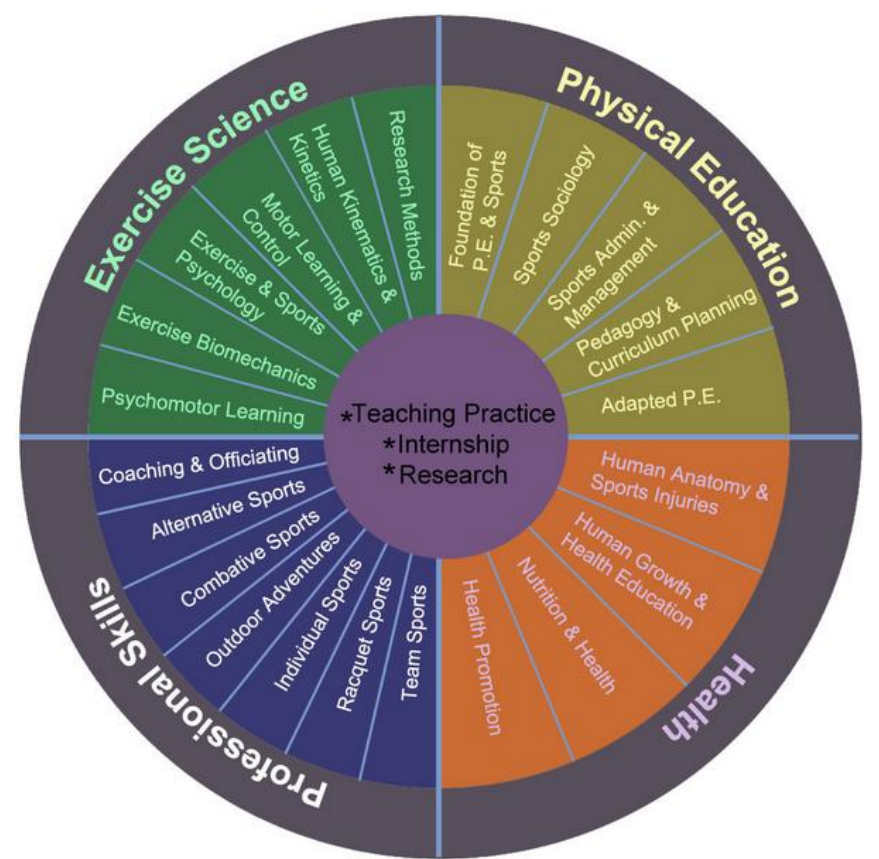

Figure 2. The Basic Components of the Physical Exercise System

Physical exercise helps to cultivate strong will quality. To engage in physical exercise to overcome difficulties, objective and subjective difficulties to overcome, good character formed in overcoming difficulties and shows. Persistent physical exercise itself is an act of will. In the process of exercise, to develop their own potential, to improve their exercise capacity at same time, also ability development of their own will. Psychological research shows that the formation of personality and its development is closely connected with the man's activities. People take part in physical exercise, can display their talents, and to realize their psychological satisfaction, achieve this mindset can enhance the people's self-esteem, self-confidence and a sense of pride to improve self-concept. Studies show that often take part in physical activity are more likely to develop the close relationships with others as interpersonal relationship more harmonious, more help to create affinity, eliminate loneliness, fear psychology, etc. and the physical exercise can strengthen the body self-esteem and self-concept.

The Hygiene of Physical Training. Basic knowledge of sports health care is an important part of the university sports theory teaching, contact practice is one of the highest degree in content. But, at present our country ordinary university sports theory class teaching shortcomings of teaching time, teaching content choice has the one-sidedness, textbooks used human factors have a larger impact, monotonous teaching form, teaching methods and means lag behind, negative problems such as the students' sports attitude. Basic knowledge of sports health care has strong theoretical and knowledge, physical education teaching in colleges and universities have leading role and students' extracurricular physical exercise steps [6-7].

Through the basic knowledge of sports health care course teaching, students can master the basic knowledge of sports and sports health knowledge, improve the understanding of the physical training purpose and meaning, to strengthen the understanding of the practice teaching content, arouse the enthusiasm of students in class and take part in the physical exercise; But also in accordance with the sports knowledge and scientific principles to exercise the body, and combine the relevant theoretical knowledge and sports practice, improve the practice of the specific aim, to overcome the blindness, strengthen college students' sports 
culture, the development of their sports intelligence and cognitive ability, better finish the task of school physical education, and form good habits of sports and exercise consciousness. Some necessary knowledge of sports health care, are the college students better take exercise, enhance physical fitness, the important guarantee to improve motor skills. Through in the process of sports teaching experience and interview of the university students found that: the modern college students' physical health knowledge is obviously insufficient their health, medical supervision of physical education and sports injury knowledge lack the necessary understanding, serious impact on their daily physical exercise and the effect of exercise that will make the comprehensive quality education affected by the certain degree.

The Sports Medicine. With the continuous development of the medical science and technology, people awareness of health more and more profound, also more and more high to the requirement of prevention and health care, and at the same time promoting public fitness activities. The continuous development of the undertakings of physical culture and sports not only the competitive sports, sports science and the technology. Sports medicine is to study how to improve the people's physical fitness exercise with the prevention and cure the problems, and provides scientific theoretical basis, use of modern medical science methods, evaluation of athlete's training, to ensure proper training athletes, prompting them to eliminate fatigue and prevent excessive fatigue, to play to the largest sports skills, improve the level of sport technology to prevent athletic injury and motility disease [8].

Accordingly, the corresponding approaches could be summarized as the follows. (1) The medical sports is an important part of the combination therapy for many diseases, is an important means of promoting or restoring skills. Experience has shown that although the wounded in clinic has returned to state, in order to make the injured a speedy recovery exercise capacity, it is necessary to use health sports. (2) Medical treatment is the combination of sport and medicine dialectical, and applied to the clinical a cross edge discipline. Medical sports can play the treatment and prevention of two kinds of effect that can enhance physical fitness, and can prevent the happening of the disease.

Health is not only refers to the absence of disease or illness, but also a kind of physical, mental and social completely in good condition. Although clinical medicine has played an important role in the aspect of saving human life, but the appeals of the promotion to the health of people can't fully satisfy, show some limitations and the passivity. Sports as important health method has always paid the great attention for the people, its positive role also to health is becoming more and more attention, and especially in the field of health and rehabilitation medicine.

\section{Conclusion}

In this paper, we conduct research on the sunlight sports and the corresponding involved medical problems and sports health knowledge. It is the purpose of the college students to participate in the physical exercise to enhance physical fitness, but insist for a long time to take part in physical exercise by the school sports teaching content boring, the restriction of such factors as the lack of the space equipment, affect the students' enthusiasm to take part in physical exercise. The interest is a part of the exercise form physical exercise habit of the foundation. College offers students like sports, meet the students desire to participate in the activities to arouse students' interest in participating in physical exercise, good motivated students to take an active part in physical exercise, to form the habit of the physical exercise. In the future, we will conduct more in-depth analysis for optimization. 


\section{References}

[1] Zhen, G. U. O. "The Present Situation and Countermeasures of College Sports Teaching under the Background of Sunlight Sports." Yinshan Academic Journal (Natural Science Edition) 4 (2012): 031.

[2] LIU, Xiao-hui, and Sheng PAN. "A Comparative Study on the Implementation Process and the Effectiveness Evaluati on of Sunlight Sports in Colleges and Middle Schools in Guangdong Province." Journal of Hebei Institute of Physical Education 4 (2012): 015.

[3] ZHANG, Xu-hong, and Lan-fang ZENG. "The development sunlight sports meet question and countermeasure research." Journal of Hubei Radio \& Television University 2 (2012): 078.

[4] LIU, Hai-jun, et al. "Implementation Status of Rural Middle School in Zhao Tong City
Sunshine Sports." Natural Science Journal of Hainan University 1 (2013): 013.

[5] Ruihua, C. A. O. "Tradition and Reform of PE Teaching in Colleges for Nationalities." (2013): 015.

[6] Ping, G. U. "Zhongshan Vocational Institute of Technology to Carry out Investigation and Analysis of the Sunshine Sports." Journal of Nanjing Institute of Physical Education (Social Science) 2 (2012): 021.

[7] Nesa, Badrun, et al. "Algal symbionts increase DNA damage in coral planulae exposed to sunlight." Zoological Studies 51 (2012): 12-17.

[8] Desbrow, Ben, et al. "Sports dietitians Australia position statement: Sports nutrition for the adolescent athlete." International Journal of Sports Nutrition and Exercise Metabolism (2014). 